\title{
THE INTERFACES OF ONE-DIMENSIONAL FLOWS IN POROUS MEDIA
}

\author{
BY \\ JUAN L. VÁZQUEZ ${ }^{1}$
}

\begin{abstract}
The solutions of the equation $u_{t}=\left(u^{m}\right)_{x x}$ for $x \in \mathbf{R}, 0<t<$ $T, m>1$, where $u(x, 0)$ is a nonnegative Borel measure that vanishes for $x>0$ (and satisfies a growth condition at $-\infty$ ), exhibit a finite, monotone, continuous interface $x=\varsigma(t)$ that bounds to the right the region where $u>0$. We perform a detailed study of $\zeta$ : initial behaviour, waiting time, behaviour as $t \rightarrow \infty$. For certain initial data the solutions blow up in a finite time $T^{*}$ : we calculate $T^{*}$ in terms of $u(x, 0)$ and describe the behaviour of $\zeta$ as $t \uparrow T^{*}$.
\end{abstract}

Introduction. We consider the initial-value problem for the porous media equation

$$
\begin{cases}u_{t}=\left(u^{m}\right)_{x x} & \text { in } Q_{T}=\mathbf{R} \times(0, T), 0<T \leq \infty, \\ u(x, 0)=u_{0}(x) & \text { for } x \in \mathbf{R},\end{cases}
$$

where $m>1$ is a physical constant and $u_{0}$ satisfies the assumptions $u_{0}$ is a nonnegative Borel measure in $\mathbf{R}, u_{0} \not \equiv 0$,

$$
\sup _{R \geq 1} R^{-(m+1) /(m-1)} \int_{|x| \leq R} d u_{0}(x)<\infty,
$$

$u_{0}$ vanishes on $(0, \infty)$.

The equation appears in a number of applications, the most typical being the flow of gas through a porous medium, where $u$ stands for the density of the gas. This motivates the assumption (H1). Assumption (H2) is justified in view of the existence theory: in fact Bénilan, Crandall and Pierre [9] have constructed continuous weak solutions to the $N$-dimensional analogue: $\left(\mathrm{P}_{N}\right) u_{t}=\Delta u^{m}, u(x, 0)=u_{0}(x), N \geq 1$, in a maximal strip $Q_{T^{*}}=\mathbf{R}^{N} \times\left(0, T^{*}\right), 0<T^{*}=T^{*}\left(u_{0}\right) \leq \infty$, under the condition

$$
\sup _{R \geq 1} R^{-(N+2 /(m-1))} \int_{|x| \leq R} d\left|u_{0}\right|(x)<\infty
$$

that reduces to (H2) if $N=1, u_{0} \geq 0$. Whenever $T^{*}<\infty$ we say that the solution blows up in a finite time. They also prove that for nonnegative solutions a necessary and sufficient condition for global existence, i.e. $T^{*}=\infty$, is

$$
\lim _{R \rightarrow \infty} R^{-(N+2 /(m-1))} \int_{|x| \leq R} d u_{0}(x)=0 .
$$

Received by the editors July 15, 1983.

1980 Mathematics Subject Classification. Primary 35K65, 76S05, 35B40.

Key words and phrases. Flows in porous media, interfaces, blow-up time, waiting time, asymptotic behaviour.

${ }^{1}$ This work was done while the author was a Fulbright Scholar 1982-1983. Sponsored by the United States Army under Contract No. DAAG29-80-C-0041.

(C) 1984 American Mathematical Society $0002-9947 / 84 \$ 1.00+\$ .25$ per page 
Also Aronson and Caffarelli [4] showed that every continuous, nonnegative weak solutions of $u_{t}=\Delta u^{m}$ in a strip $Q_{T}, T>0$, has an initial trace $u(x, 0)$ which is a locally bounded measure satisfying the growth condition ( $\left.\mathrm{H} 2^{\prime}\right)$.

Recently Dahlberg and Kenig $[\mathbf{1 1}]$ proved that the continuous, nonnegative distributional solutions of $\left(\mathrm{P}_{N}\right)$ in $Q_{T}, T>0$, are unique. For another uniqueness result cf. [9] and its references. Early work on this subject goes back to Kalashnikov $[14]$.

In view of these results (H2) is an optimal growth condition for the initial values of $(\mathrm{P})$.

In this paper we are interested in describing the free-boundaries that appear in (P): indeed one of the most appealing features of $(\mathrm{P})$ with $m>1$ is the fact that when $u_{0}$ vanishes outside a compact interval then the support of $u(\cdot, t), t>0$, is also compact. This is called the finite propagation property and has been described by Oleinik, Kalashnikov and Czhou [17] in their 1958 paper where the existence, uniqueness, regularity and finite propagation for the solutions of $(\mathrm{P})$ were first discussed at length.

The above considerations lead us to introduce the assumption (H3). We show that the solutions of $(\mathrm{P})$ under the assumptions (H1)-(H3) vanish for large enough $x \geq 0$ for any fixed time $0<t<T^{*}$. We define the outer right interface (or free boundary) of $u$ as the curve $x=\varsigma(t)$, where

$$
\left\{\begin{array}{l}
\varsigma(t)=\sup \{x: u(x, t)>0\} \quad \text { if } 0<t<T^{*}, \\
\varsigma(0)=\sup \left\{x: \int_{(x, \infty)} d u_{0}>0\right\} .
\end{array}\right.
$$

Without loss of generality we may assume that $\zeta(0)=0$.

Under the additional conditions that $u_{0}$ is continuous and has compact support, many properties of the function $t \rightarrow \varsigma(t)$ are known, cf. $[\mathbf{1}, \mathbf{6}, \mathbf{1 6}, \mathbf{1 8}, \mathbf{2 0}]: \varsigma(t)$ is a continuous, nondecreasing function of $t, 0 \leq t<\infty$, and there exists a time $t^{*}, 0 \leq t^{*}<0$, called waiting time such that $\zeta(t)=\varsigma(0)$ if $0<t<t^{*}$ and $\varsigma \in C^{1}\left(t^{*}, \infty\right)$ and $\varsigma^{\prime}(t)>0$ for $t>t^{*}$. Moreover, the following equation is satisfied at the interface $[\mathbf{1}, \mathbf{1 6}]$ :

$$
\varsigma^{\prime}(t)=-\left(m u^{m-1} /(m-1)\right)_{x}(\varsigma(t), t),
$$

and the interface shows a certain convexity $[\mathbf{6}, \mathbf{2 0}]$

$$
\varsigma^{\prime \prime}(t) \geq-\frac{m}{(m+1) t} \zeta^{\prime}(t)
$$

(here $\zeta^{\prime \prime}$ is understood as a measure). In [20] initial data satisfying $u_{0} \in L^{1}(\mathbf{R}), u_{0}$ $\geq 0$ a.e., and (H3) are considered and the asymptotic behavior of $\zeta(t)$ as $t \rightarrow \infty$ is studied.

This paper studies the interfaces $\zeta:\left[0, T^{*}\right) \rightarrow[0, \infty)$ that occur when we consider solutions $u$ of $(\mathrm{P})$ with general initial data, that is, under the sole assumptions (H1), (H2), (H3). Thus in $\S 1$ we show that the above results remain valid for our general solutions; now $0 \leq t<\infty$ must be replaced by $0 \leq t<T^{*}$.

The rest of the paper is devoted to the study of the following properties of $\zeta(t)$ : behaviour for $t \cong 0$, existence of a positive waiting time, existence or nonexistence 
of a blow-up in finite time, behaviour of $\varsigma(t)$ as $t \rightarrow \infty$ if $T^{*}=\infty$ or as $t \rightarrow T^{*}$ if $T^{*}$ is finite.

The basic technique used throughout the paper is comparison with suitable selfsimilar solutions via a Shifting-Comparison Principle (introduced by the author in [20]).

In the next paragraphs we present our main results under some simplifying assumptions that allow for a clearer picture. We assume to begin with that $u_{0} \in$ $L_{\text {loc }}^{1}(\mathbf{R})$ and let $p_{0}=\left(m u_{0}^{m-1} /(m-1)\right)$. In the application to the flow of gas through a porous medium $p_{0}$ is the pressure of the gas at $t=0$.

In $\S 2$ we estimate the blow-up time $T^{*}$ in terms of the growth of $u_{0}$ as $x \rightarrow-\infty$. Thus if

$$
p_{0}(x) / x^{2} \rightarrow \alpha \text { as } x \rightarrow-\infty, 0 \leq \alpha<\infty
$$

we have

$$
\frac{1}{2(m+1) \alpha} \leq T^{*} \leq \frac{\mu}{2(m+1) \alpha}
$$

$(1 / 0=\infty)$ for a certain constant $\mu>0$ that depends only on $m$; cf. Theorem 1 for the general result.

$\S 3$ is devoted to a similar study of the waiting time $t^{*}$ : we obtain a necessary and sufficient condition on $u_{0}$ for a positive waiting time to exist: $t^{*}>0$ if and only if

$$
\limsup _{x \rightarrow 0}|x|^{-(m+1) /(m-1)} \int_{(x, 0]} d u_{0}(\xi)<\infty .
$$

Remark that (0.7) only depends on the behaviour of $u_{0}$ near $x=0$. Estimates for $t^{*}$ are also obtained in Theorem 2 .

To study the behaviour of $\zeta(t)$ as $t \rightarrow 0$ if $t^{*}=0$ or as $t \rightarrow \infty$ if $T^{*}=\infty$ we need suitable examples of solutions with which to compare. They are the self-similar solutions of $(\mathrm{P})$ with initial data $u_{0}(x)=(\alpha+1)|x|^{\alpha}, 2 /(m-1)>\alpha>-1$, that we study in $\S 4$.

By means of our comparison technique we obtain in $\S 5$ results about the limit $t \downarrow 0$; if $v_{0}(x) \sim|x|^{\lambda}$ as $x \rightarrow 0,2>\lambda>-(m-1)$, then $\zeta(t) \sim t^{\gamma}$ as $t \rightarrow \infty$ with $\gamma=1 /(2-\lambda)$. A completely analogous result holds for $\varsigma(t)$ as $t \rightarrow \infty$ if $u_{0}(x) \sim|x|^{\lambda}$ as $x \rightarrow-\infty$. This is done in $\S 6$.

Finally in $\S 7$ we study the behaviour of $\varsigma(t)$ as $t \rightarrow T^{*}$ when $T^{*}$ is finite. In particular it may happen that $\zeta(t)$ exhibits an asymptote; that is, $\varsigma(t) \uparrow \infty$ as $t \uparrow T^{*}$. We also study the blow-up set, i.e. the set of points $x \in \mathbf{R}$ for which $u(x, t) \rightarrow \infty$ as $t \rightarrow T^{*}$. Both phenomena are described in terms of $u_{0}$.

An interesting question not dealt with here is the optimal regularity of $\zeta$. As we said above, $\zeta(t)$ is a $C^{1}$ function for $0<t<T^{*}$ unless maybe at $t=t^{*}$ when $0<t^{*}<T^{*}$. In [5] Aronson, Caffarelli and Kamin exhibited a class of initial data for which $\zeta$ is $C^{1}$ even at $t=t^{*}$; see also [15]. Nevertheless not all the solutions of $(\mathrm{P})$ have a $C^{\mathbf{1}}$ smooth interface: recently Aronson, Caffarelli and the author [6] have proved that for a class roughly complementary to the one in [5] the solutions start abruptly after a positive waiting time: $\zeta^{\prime}\left(t^{*}+\right)>0$. We refer to [6] for further details. 


\section{Preliminaries.}

1.1. Existence and uniqueness of solutions. We recall here the results that we need from [9]:

THEOREM A. Let $u_{0}$ be a Borel measure satisfying (H2). Then there exists a maximal time $T^{*} \in(0, \infty]$ for which a solution $u$ can be defined in $\left(0, T^{*}\right)$ such that:

(i) $u \in C\left(\left(0, T^{*}\right) ; L_{\text {loc }}^{1}(\mathbf{R})\right) \cap L_{\text {loc }}^{\infty}\left(\left[0, T^{*}\right) ; X\right)$.

(ii) $u(x, t)\left(1+|x|^{2}\right)^{-1 /(m-1)} \in L_{\mathrm{loc}}^{\infty}\left(\mathbf{R} \times\left(0, T^{*}\right)\right)$.

(iii) For $\psi \in C_{0}^{\infty}\left(\mathbf{R} \times\left[0, T^{*}\right)\right)$ we have

$$
\int_{0}^{T^{*}} \int\left(u \psi_{t}+u^{m} \psi_{x x}\right) d x d t+\int \psi(x, 0) d u_{0}(x)=0 .
$$

Moreover, if $T^{*}<\infty$ (in which case we say that the solution blows up in finite time)

(iv) $\lim _{t \uparrow T^{*}}\left|\|u(\cdot, t) \mid\|_{1}=\infty\right.$.

Here and above $X$ denotes the space of functions $f \in L_{\text {loc }}^{1}(\mathbf{R})$ such that

$$
\left\||| f\left|\|_{r}=\sup _{R \geq r} R^{-(m+1) /(m-1)} \int_{|x| \leq R}\right| f \mid d x<\infty\right.
$$

for some (= all) $r>0$, equipped with the norm $\||\cdot|\|_{1} \cdot[9]$ contains further information on the solutions: uniqueness,.... In particular, it is important to remark that the solution $u$ with initial data $u_{0} \geq 0$ can be obtained as the limit of solutions $u_{n}$ with smooth, compactly supported initial data. Also P. Sacks proved that $u$ is continuous in $Q_{T^{*}}[\mathbf{1 9}]$.

For uniqueness and comparison purposes we shall use the following result of Dahlberg and Kenig [11].

THEOREM B. Let $u_{1}(x, t), u_{2}(x, t)$ be continuous, nonnegative functions in a strip $Q_{T}=\mathbf{R} \times(0, T), T>0$, such that

(i) $u_{1}$ and $u_{2}$ are solutions of $u_{t}=\left(u^{m}\right)_{x x}$ in $D^{\prime}\left(Q_{T}\right)$;

(ii) the initial traces $u_{1}(x, 0), u_{2}(x, 0)$ (that exist thanks to [4]) satisfy $u_{1}(x, 0) \leq$ $u_{2}(x, 0)$ as measures.

Then $u_{1}(x, t) \leq u_{2}(x, t)$ in $Q_{T}$.

1.2. Properties of the solutions. The following properties are valid for solutions with smooth initial data in $L^{1}(\mathbf{R})$ and remain valid for general initial data by approximation.

PROPERTY S1. (i) $\left(u^{m-1}\right)_{x x} \geq-(m-1) / m(m+1) t$ and (ii) $u_{t} \geq-u /(m+1) t$ in $D^{\prime}\left(Q_{T^{*}}\right)$.

PROPERTY S2. If $u_{0}$ is a function such that $\left(u_{0}^{m-1}\right)_{x x} \geq 0$ in $D^{\prime}(\mathbf{R})$, then $\left(u(x, t)^{m-1}\right)_{x x} \geq 0$ in $D^{\prime}(\mathbf{R})$ for every $t>0$.

PROPERTY S3. Given two solutions $u, \hat{u}$ with initial data $u_{0}, \hat{u}_{0}$ we have, for every $t>0$ for which both are defined,

$$
\int(u(x, t)-\hat{u}(x, t))_{+} d x \leq \int\left(d u_{0}(x)-d \hat{u}_{0}(x)\right)_{+}
$$

where $(\cdot)_{+}=\max (\cdot, 0)$. 
We remark that Property S3 implies, in particular, the pointwise comparison result; cf. [3] for Property S1, [1, Lemma 2] for Property S2 and [8, 9] for Property S3. For the next property we refer to our work [20].

PROPERTY S4 (SHIFTING-COMPARISON LEMMA). Let $u, \hat{u}$ be solutions of (P) under conditions (H1)-(H3). If $u_{0}, \hat{u}_{0}$ satisfy

$$
\int_{x}^{\infty} d u_{0}(x) \leq \int_{x}^{\infty} d \hat{u}_{0}(x)
$$

for every $x \in \mathbf{R}$, then for every $t>0$ where both are defined we have

$$
\int_{x}^{\infty} u(x, t) d x \leq \int_{x}^{\infty} \hat{u}(x, t) d x .
$$

COROLLARY S1. Under the above assumptions, if $\varsigma(t), \hat{\zeta}(t)$ are the interfaces defined in (0.2) and $t>0$ is as above, then

$$
\zeta(t) \leq \hat{\zeta}(t) .
$$

Notation. We shall use the notation $u_{0} \prec \hat{u}_{0}$ or $\hat{u}_{0} \succ u_{0}$ meaning that (1.3) holds. Conclusion (1.4) is then written as $u(\cdot, t) \prec \hat{u}(\cdot, t)$.

1.3. Some explicit solutions. The following solutions will play an important role in the sequel as the models with which we compare other solutions. First we consider the solutions $w(x, t ; M)$ of $(\mathrm{P})$ with $w(x, 0 ; M)=M \delta(x)$, where $M>0$ and $\delta$ is Dirac's delta function. They are given by

$$
w(x, t ; M)=t^{-1 /(m+1)}\left(C-\frac{m-1}{2(m+1) m} \cdot \frac{x^{2}}{t^{2 /(m+1)}}\right)_{+}^{1 /(m-1)}
$$

(cf. [7]), where $C$ and $M$ are related by

$$
M=a_{m} C^{(m+1) / 2(m-1)}, \quad \text { with } a_{m}=\left(\frac{2 m(m+1)}{m-1}\right)^{1 / 2} B\left(\frac{m}{m-1}, \frac{1}{2}\right)^{2}
$$

The right interface of $w(x, t ; M)$ is given by $x=r(t)$, where

$$
r(t)=\left(\frac{2 m(m+1)}{m-1} C\right)^{1 / 2} t^{1 /(m+1)}=c_{m} M^{(m-1) /(m+1)} t^{1 /(m+1)},
$$

with $c_{m}=(2 m(m+1) /(m-1))^{1 /(m+1)} \cdot B(m /(m-1), 1 / 2)^{-(m-1) /(m+1)}$.

The solutions $w(x, t ; M)$ serve as a model of solutions with $L^{1}$-data; $\mathrm{cf}$. [20]. For solutions that blow up in finite time we shall use as model the family $z(x, t ; T, C)$ defined in $Q_{T}, T>0$, by

$$
z(x, t ; T, C)=(T-t)^{-1 /(m+1)}\left(\frac{m-1}{2 m(m+1)} \cdot \frac{x^{2}}{(T-t)^{2 /(m-1)}}+C\right)_{+} .
$$

$C$ can be any real number. If $C>0, z$ is always positive. If $C<0, z$ vanishes in the region $|x| \leq r(T-t)$ with $r$ defined as in (1.8). In this case we can consider the restrictions

$$
z_{-}(x, t ; T, C)=z(x, t ; T, C) H(-x),
$$

\footnotetext{
${ }^{2} B(\cdot, \cdot)$ is Euler's beta function.
} 


$$
z_{+}(x, t ; T, C)=z(x, t ; T, C) H(x)
$$

where $H(x)=1$ if $x \geq 0, H(x)=0$ if $x<0$. If $C \leq 0, z_{+}, z_{-}$are solutions of (P) and, in fact, the right-interface of $z_{-}$is the curve $x=-r(T-t), 0<t<T$.

We shall write $z(x, t ; T), z_{ \pm}(x, t ; T)$ instead of $z(x, t ; T, 0), z_{ \pm}(x, t ; T, 0)$.

1.4. Properties of the interface. Let $u$ be the solution of $(\mathrm{P})$ under conditions $(\mathrm{H} 1),(\mathrm{H} 2),(\mathrm{H} 3)$ and let $\zeta(t)$ be its interface as in $(0.2)$. We have

PROPERTY I1. $\varsigma(t)$ is finite and nondecreasing for $0<t<T^{*}$.

PROOF. It is nondecreasing since Property S1(ii) implies that if $u(x, t)>0$ and $\bar{t}>t$, then $u(x, \bar{t})>0$.

To see that it is finite we remark that by Properties (H2), (H3) there exist constants $C_{1}, C_{2}>0$ such that

$$
\int_{x}^{\infty} d u_{0}(x) \leq c_{1}\left(|x|+c_{2}\right)^{(m+1) /(m-1)}
$$

hence there exists $T_{1}>0$ such that $u_{0} \prec z_{-}\left(x-C_{2}, 0 ; T_{1}\right)$ and the ShiftingComparison Lemma implies that

$$
\varsigma(t) \leq C_{2} \text { for } 0<t<T_{1} .
$$

It is clear from Theorem $\mathrm{A}(\mathrm{iv})$ that $T^{*} \geq T_{1}$. In case $T^{*}>T_{1}$ we can repeat the argument above up to any time $T<T^{*}$ using the fact $u \in L_{\text {loc }}^{\infty}\left(\left[0, T^{*}\right), X\right)$.

We can now define the waiting time $t^{*}$ as in the introduction. We have $\zeta(t)=0$ if $0 \leq t \leq t^{*}$ and $\zeta(t)>0$ if $t^{*}<t<T^{*}$. We shall show in $\S 3$ that $t^{*}$ is finite. We recall that the local velocity of a solution is defined in the set $\{(x, t): u(x, t)>0\}$ by $V(x, t)=-\left(m u^{m-1} /(m-1)\right)_{x}$; cf. e.g. [1]. If $t^{*}<T^{*}$ we have

PROPERTY I2. $\varsigma \in C^{1}\left[t^{*}, T^{*}\right)$ and for $0<t<T^{*}$ the limit

$$
\lim _{\substack{x \rightarrow \zeta(t) \\ u(x, t)>0}} V(x, t)=V(\varsigma(t), t)
$$

exists and equals $\varsigma^{\prime}(t+)$ if $t \geq 0$. Moreover,

$$
\varsigma^{\prime \prime}(t)+\frac{m}{(m+1) t} \zeta^{\prime} \geq 0 \text { in } D^{\prime}\left(t^{*}, T^{*}\right),
$$

therefore $\zeta^{\prime}(t) t^{m /(m+1)}$ is nondecreasing and $\zeta^{\prime}(t)>0$ if $t>t^{*}$.

PROOF. (1.14) was proved in [1] and [16] for solutions with continuous, compactly supported initial data; $\varsigma \in C^{1}$ is proved in [10] and for (1.15) cf. [10] and [20]. The essential of the proof remains unchanged using the properties aleady quoted and the remark that Theorem A(ii) and Property S1(i) imply that, for every $0<t<T^{*}, V(x, t)$ is a locally bounded function of $x$.

PROPERTY I3. If $u_{0}$ is a function such that $\left(u_{0}^{m-1}\right)_{x x} \geq 0$ in $D^{\prime}(\mathbf{R})$, then $\varsigma(t)$ is a convex function of $t, 0<t<t^{*}$.

ProOF. By Property S2, $V(x, t)$ is a nondecreasing function of $x$ for every $t>0$. This means that $V(x, \bar{t}) \geq V(\varsigma(\bar{t}), \bar{t})=k \geq 0$ : therefore, if $k>0$, the "constant-velocity front"

$$
\bar{u}=\left(\frac{m-1}{m} k[k(t-\bar{t})-(x-\varsigma(\bar{t}))]_{+}\right)^{1 /(m-1)}
$$


is a solution of $(\mathrm{P})$ in $\mathbf{R} \times\left(\bar{t}, T^{*}\right)$ such that $\bar{u} \leq u$. Hence for every $t>\bar{t}$

$$
\varsigma(t) \geq \varsigma(\bar{t})+\varsigma^{\prime}(\bar{t})(t-\bar{t}) .
$$

This means that $\zeta$ is convex. We remark that when $\bar{t}=t^{*}$ we take $\varsigma^{\prime}(\bar{t})$ to mean $\varsigma^{\prime}(\bar{t}+)$.

2. Blow-up time. In this section we are concerned with the blow-up of the solutions of $(\mathrm{P})$. We shall ask that $u_{0}$ satisfies (H1), (H2). Since (H3) plays no significant role it is dropped in this section. As we said before it has been proved in [9] that the blow-up time $T^{*}$ of a solution $u$ is finite if and only if the quantity

$$
L_{0}=L\left(u_{0}\right) \equiv \limsup _{|x| \rightarrow \infty}|x|^{-(m+1) /(m-1)} M(x)
$$

is positive, where $M(x)=\int_{I} d u_{0}(\xi)$ is the mass of $u_{0}$ contained in the interval $I=(x, 0]$ if $x<0, I=[0, x)$ if $x>0$. $L_{0}$ measures the growth of $u_{0}$ as $|x| \rightarrow \infty$ and is finite and nonnegative by (H1), (H2).

Here we obtain precise bounds for $T^{*}$ in terms of $L_{0}$. In doing this we first meet the comparison technique that is used throughout this paper.

We begin by showing that $T^{*}$ depends only on the behaviour of $u_{0}$ for very large $|x|$ : for any $a \in \mathbf{R}$ we define $u_{a}^{1}$ as the solution of $(\mathrm{P})$ with initial value $u_{0}^{1}(x ; a)=u_{0}(x) \cdot \chi((-\infty, a])$, i.e. $u_{0}^{1}(x ; a)$ coincides with $u_{0}$ on $(-\infty, a]$ and vanishes on $(a, \infty)$. Likewise we define $u_{b}^{2}$ for some $b \in \mathbf{R}$ as the solution of (P) with initial value $u_{0}^{2}(x ; b)=u_{0}(x) \cdot \chi([b, \infty))$. Then we have

PROPOSITION 2.1. All the solutions $u_{a}^{1}, a \in \mathbf{R}$, have the same blow-up time $T_{1}^{*}$. Likewise the family $\left\{u_{b}^{2}\right\}$ has a common blow-up time $T_{2}^{*}$. Finally

$$
T^{*}(u)=\min \left(T_{1}^{*}, T_{2}^{*}\right) .
$$

ProOF. By the maximum principle (cf. (1.2)) we have, for every $a^{\prime}<a, b<b^{\prime}$,

$$
\begin{aligned}
& T^{*}(u) \leq T^{*}\left(u_{a}^{1}\right) \leq T^{*}\left(u_{a^{\prime}}^{1}\right), \\
& T^{*}(u) \leq T^{*}\left(u_{b}^{2}\right) \leq T^{*}\left(u_{b^{\prime}}^{2}\right) .
\end{aligned}
$$

The fact that $T^{*}\left(u_{a}^{1}\right)=T^{*}\left(u_{a^{\prime}}^{1}\right)$ is a consequence of (1.2) and Theorem $\mathrm{A}$ (iv): (1.2) implies that, for $t<T^{*}\left(u_{a}^{1}\right), u_{a}^{1} \geq u_{a^{\prime}}^{1}$ and $\int\left(u_{a}^{1}-u_{a^{\prime}}^{2}\right)_{+} d x$ is bounded by a constant that does not depend on $t$. Therefore, \|\|$u_{a}^{1}(\cdot, t)\|\|_{1}$ is bounded as long as $\left\|\mid u_{a^{\prime}}^{1}(\cdot, t)\right\|_{1}$ is. By virtue of Theorem A(ii), (iv) this implies that $T^{*}\left(u_{a}^{1}\right)=T^{*}\left(u_{a^{\prime}}^{1}\right)$.

The same argument proves that $T^{*}\left(u_{b}^{2}\right)=T^{*}\left(u_{b^{\prime}}^{2}\right)$.

To end the proof we have to show that if $T=\min \left(T_{1}^{*}, T_{2}^{*}\right)$, then $u$ is defined for $0<t<T$. For this we take an $\varepsilon>0$ and prove that the supports of $u_{a}^{1}(\cdot, t)$ and $u_{b}^{2}(\cdot, t)$ do not meet for $0<t<T-\varepsilon$ if $a \ll 0$ and $b \gg 0$. Assuming that this is true we conclude as follows: $\bar{u}(x, t)=u_{a}^{1}(x, t)+u_{a}^{2}(x, t)$ is then the solution to $(\mathrm{P})$ in the domain $Q_{T-\varepsilon}$ with initial data $u_{0}-u_{0} \cdot \chi((a, b))$. From (1.2) we deduce that $u(x, t)$ is defined in $Q_{T-\varepsilon}$ and that

$$
\int u(x, t) d x \leq \int \bar{u}(x, t) d x+\int_{(a, b)} d u_{0}(x)
$$

for any $0<t<T-\varepsilon$. Now let $\varepsilon \rightarrow 0$ to get $T^{*}(u) \geq T$. 
We control finally the supports of $u_{a}^{1}$ and $u_{b}^{2}$. Let us begin with $u_{a}^{1}$ : by Theorem A(i) there exists a constant $C>0$ (that depends on $\varepsilon$ ) such that, for every $a<$ $0,0<t<T-\varepsilon$ and $x<-1$,

$$
\int_{x}^{\infty} u_{a}^{1}(x, t) d s \leq C|x|^{(m+1) /(m-1)} .
$$

Now we observe that if we set $v_{a}(x, t)=z_{-}(x-a / 2, t ; \tau)$ with

$$
\tau=\left(\frac{m-1}{m+1}\right)^{m}(8 m C)^{-1}
$$

then (2.5) implies that $u_{a}^{1}(x, 0) \prec v_{a}(x, 0)$ for every $a<-1$ so that Corollary S1 of $\S 2$ implies that $u_{a}^{1}(x, t)=0$ for every $x \geq a / 2$ and $0<t \leq \min (\tau, T-\varepsilon)$. Now let $N$ be the least integer $\geq(T-\varepsilon) / \tau$ and set $\bar{u}=u_{a^{\prime}}^{1}$ with $a^{\prime}=-2^{N}$. If $T-\varepsilon \leq \tau, N=1$ and we have proved that $\bar{u}(x, t)$ vanishes in $(-1, \infty) \times(0, T-\varepsilon)$. If $T-\varepsilon>\tau$ we can repeat the argument at $t=\tau$ with $v=z_{-}(x-a / 4, t-\tau ; \tau)$ to conclude that $\bar{u}(x, t)=0$ in $(a / 4, \infty) \times(0, \min (2 \tau, T-\varepsilon))$. By induction it follows that $\bar{u}(x, t)=0$ for $x \geq-1$ and $0<t<T-\varepsilon$ in any case.

In the same way we can prove that $u_{b^{\prime}}^{2}$ with $b^{\prime}=2^{N^{\prime}}$ and $N^{\prime}$ defined similarly to $N$ vanishes in $(-\infty, 1) \times(0, T-\varepsilon)$. This completes the proof.

The preceding result allows us to reduce the study of the blow-up time to solutions satisfying (H1), (H2), (H3). In this case

$$
L_{0}=\limsup _{x \rightarrow-\infty}|x|^{-(m+1) /(m-1)} \int_{(x, \infty)} d u_{0}(x) .
$$

The main result of this section is

THEOREM 1. $T^{*}$ is infinite if and only if $L_{0}=0$. If $L_{0}>0$ we have

$$
T_{m} / L_{0}^{m-1} \leq T^{*} \leq \theta_{m} / L_{0}^{m-1},
$$

where

$$
T_{m}=\left(\frac{m-1}{m+1}\right)^{m} \cdot \frac{1}{2 m} \quad \text { and } \quad \theta_{m}=c_{m}^{-(m+1)}=\frac{m-1}{2 m(m+1)} \cdot B\left(\frac{m}{m-1}, \frac{1}{2}\right)^{m-1}
$$

(cf. (1.9)). In case $L_{0}$ is actually the limit as $x \rightarrow-\infty$ or as $x \rightarrow \infty$ then

$$
T^{*}=T_{m} / L_{0}^{m-1} \text {. }
$$

ProOF. By virtue of Proposition 2.1 we can assume that $u_{0} \equiv 0$ on $(0, \infty)$.

Let $\varepsilon>0$. There exists a constant $C=C_{\varepsilon}>0$ such that

$$
M(x) \leq\left(L_{0}+\varepsilon\right)|x|^{(m+1) /(m-1)} \quad \text { if } x<-C .
$$

Therefore there exists a constant $K \geq 0$ such that $u_{0}(x) \prec z_{-}\left(x-K, 0 ; T_{\varepsilon}\right)$, where $T_{\varepsilon}=T_{m}\left(L_{0}+\varepsilon\right)^{1-m}$. It follows then from Property S4 that $T^{*} \geq T_{\varepsilon}$ (and that for every $\left.0<t<T_{\varepsilon}, u(\cdot, t) \prec z_{-}\left(\cdot-K, t ; T_{\varepsilon}\right)\right)$. Letting $\varepsilon \rightarrow 0$ we obtain the left-hand inequality of $(2.8)$.

In case $L_{0}$ is not only a lim sup but the limit as $x \rightarrow-\infty$ of the expression in (2.7) we can repeat the argument now to find a $K<0$ such that $u_{0}(x) \succ$ $z_{-}\left(x-K, 0 ; T_{-\varepsilon}\right)$, where obviously $T_{-\varepsilon}=T_{m}\left(L_{0}-\varepsilon\right)^{1-m}$. It follows that $T^{*} \leq T_{\varepsilon}$; hence, as $\varepsilon \rightarrow 0, T^{*} \leq T_{m} L_{0}^{1-m}$. 
We prove next the second inequality of (2.8): we choose a point $\bar{x}<-1$, move the mass in $[\bar{x}, 0]$ at time $t=0$ to the point $\bar{x}$ and consider the solution $\bar{u}(x, t)=$ $w(x-\bar{x}, t ; M(\bar{x}))$ with initial data $\bar{u}_{0}(x)=M(\bar{x}) \delta(x-\bar{x})$ : we have for every $x \in$ $\mathbf{R}, t>0$

$$
\bar{u}(x, t)=\left(\frac{m-1}{2 m(m+1) t}\right)^{1 /(m-1)}\left[c_{m}^{2} M(\bar{x})^{2(m-1) /(m+1)} t^{2 /(m+1)}-x^{2}\right]_{+}^{1 /(m-1)} .
$$

There is a sequence $\bar{x}_{n} \rightarrow-\infty$ such that, given $\varepsilon>0$,

$$
M\left(\bar{x}_{n}\right) \geq\left(L_{0}-\varepsilon\right)\left|\bar{x}_{n}\right|^{(m+1) /(m-1)}
$$

if $n$ is large enough, $n \geq n_{\varepsilon}$. Let us set

$$
\overline{t_{\varepsilon}}=\frac{(1+\varepsilon)^{(m+1) / 2}}{c_{m}^{m+1}\left(L_{0}-\varepsilon\right)^{m-1}} ;
$$

for all large $n \geq n_{\varepsilon}$ we have

$$
c_{m} M\left(\bar{x}_{n}\right)^{(m-1) /(m+1)} t_{\varepsilon}^{1 /(m+1)} \geq(1+\varepsilon)^{1 / 2}\left|\bar{x}_{n}\right| .
$$

Hence for some $C=C_{m}>0$ we have, with $\bar{x}=\bar{x}_{n}, n \geq n_{\varepsilon}$,

$$
\bar{u}^{m-1}(x, t) \geq C\left|\bar{x}_{n}\right|^{2} \varepsilon t_{\varepsilon}^{-1}
$$

if $-1<x<0$. Since by construction we have $M\left(\bar{x}_{n}\right) \delta\left(x-x_{n}\right) \prec u_{0}(x)$ we conclude that for every $t<T^{*}, \bar{u}(\cdot, t) \prec u(\cdot, t)$ and, in particular,

$$
\int_{-1}^{\infty} u(x, t) d x \geq \int_{-1}^{0} \bar{u}(x, t) d x
$$

In case $T^{*}>t_{\varepsilon}$ for an $\varepsilon>0$ we can use (2.13) to estimate the right-hand side of (2.14) and let $n \rightarrow \infty$ to conclude that the integral $\int_{-1}^{\infty} u\left(x, t_{\varepsilon}\right) d x=\infty$, a contradiction. Hence $T^{*} \leq t_{\varepsilon}$ and letting $\varepsilon \rightarrow 0$ the result follows.

REMARK. (1) The accuracy of estimate (2.8) depends on the ratio

$$
\mu_{m}=\frac{\theta_{m}}{T_{m}}=\left(\frac{m+1}{m-1}\right)^{m-1} \cdot B\left(\frac{m}{m-1}, \frac{1}{2}\right)^{m-1} .
$$

$\mu_{m}$ approaches 1 as $m \downarrow 1$. Indeed, $\mu_{1+\varepsilon}=1+O(\varepsilon \log \varepsilon)$ as $\varepsilon \downarrow 0$. On the contrary, $\mu_{m}$ grows like $2^{m}$ as $m \rightarrow \infty \cdot \mu_{2}=4$.

(2) Both constants $T_{m}$ and $\theta_{m}$ in (2.8) are sharp since they correspond to explicit solutions.

3. On the waiting time. In the sequel, $u(x, t)$ is the solution of $(\mathrm{P})$ under assumptions (H1), (H2), (H3). Without loss of generality we set $\varsigma(0)=0$. We discuss in this section the existence of a positive waiting time and give estimates for it in terms of $u_{0}$.

In [2] Aronson constructed an example of a solution with smooth initial data having a positive waiting time that he explicitly computed. To be specific if $u_{0}^{m-1}(x)=\cos ^{2} x$ for $-\pi / 2 \leq x \leq \pi / 2, u_{0}^{m-1}(x)=0$ otherwise, he proved that $t^{*}=(m-1) / 2 m(m+1)$ and at that time the second derivative $\left(u_{0}^{m-1}(x, t)\right)_{x x}$ blows up at $x= \pm \pi / 2$. Knerr discussed in [16] (under the simplifying assumptions that $u_{0}$ is continuous, positive in a bounded interval $(a, b), a<b$, and zero outside) 
the waiting time $t^{*}$ in terms of the behaviour of $p_{0}(x)=m u_{0}^{m-1} /(m-1)$ near the endpoint $b$ : thus if $p_{0}(x) \leq c(b-x)^{2}$ for some $C>0$ and all $x$ near $b$ then $t^{*}>0$; on the contrary if $p_{0}(x) \geq C(b-x)^{\alpha}$ with $C, x$ as before and $\alpha<2$ then $t^{*}=0$.

In [5] Aronson, Caffarelli and Kamin prove the following result (adapted to our notation).

THEOREM C. Let $u$ be a solution of $(\mathrm{P})$, let $p=m u^{m-1} /(m-1)$, and assume that $u_{0} \in L_{\text {loc }}^{1}(\mathbf{R})$ and that $p_{0}(x) \equiv p(x, 0)=0$ for $x>0$. If $p_{0} x=\alpha x^{2}+o\left(x^{2}\right)$ as $x \uparrow 0$ and $p_{0}(x) \leq \beta x^{2}$ in $\mathbf{R}^{-}$for some constants $\alpha, \beta>0$, then

$$
1 / 2(m+1) \beta \leq t^{*} \leq 1 / 2(m+1) \alpha
$$

COROLlaRY A. Under the above hypotheses, if $\alpha=\beta$, then

$$
t^{*}=1 / 2(m+1) \alpha .
$$

In this section we give a necessary and sufficient condition for the existence of a positive waiting time as well as an estimate of $t^{*}$ in terms of $M(x)$. Notice that under hypothesis (H3), $M(x)=0$ if $x>0$ and

$$
M(x)=\int_{(x, 0]} d u_{0}(x) \text { if } x<0 .
$$

THEOREM 2. (I) $t^{*}$ is positive if and only if

$$
\limsup _{x \rightarrow 0} M(x)|x|^{-(m+1) /(m-1)}<\infty .
$$

(II) More precisely if $B=\sup _{x<0} M(x)|x|^{-(m+1) /(m-1)}<\infty$, then

$$
T_{m} / B^{m-1} \leq t^{*} \leq \theta_{m} / B^{m-1},
$$

where $T_{m}, \theta_{m}$ are the same constants as in Theorem 1 .

(III) If $A=\liminf _{x \uparrow 0} M(x)|x|^{-(m+1) /(m-1)}$ is positive, then

$$
t^{*} \leq T_{m} / A^{m-1} \text {. }
$$

COROLLARY 3.1. If $u_{0}$ is such that the supremum of $M(x)|x|^{-(1+m) / m}$ is obtained as the limit when $x \uparrow 0$, then

$$
t^{*}=T_{m} / B^{m-1} \text {. }
$$

REMARKS. (1) Since for $p_{0}(x)=b x^{2}$ we have

$$
M(x)=\left(\frac{m-1}{m}\right)^{1 /(m-1)} \cdot \frac{m-1}{m+1} \cdot b^{1 /(m-1)}|x|^{(m+1) /(m-1)},
$$

under the hypothesis $p_{0}(x) \leq \beta x^{2}$ the left-hand inequality of (3.5) gives precisely $(2(m+1) \beta)^{-1} \leq t^{*}$. Also Corollary $\mathrm{A}$ is implied by Corollary 3.1 , the conditions being in our case far less restrictive.

(2) For the accuracy of (3.5) see Remark (1) at the end of $\S 2$.

Proof of TheOREM 2. (II) Assume that $B<\infty$. We compare $u(x, t)$ with $\bar{u}(x, t)=z(x, t ; \tau)$. It is immediate that $u_{0} \prec \bar{u}_{0}$ if $\tau \leq T_{m} B^{1-m}$. Therefore we conclude from Theorem 1 that $T^{*} \geq \tau$ and from the Shifting-Comparison Theorem that for $0<t<\tau, \zeta(t) \leq \bar{\zeta}(t)=0$; hence $t^{*} \geq \tau$. This proves (3.5), left. 
For the upper bound in (3.5) we compare $u(x, t)$ with the solution $\tilde{u}(x, t)=$ $w(x-\bar{x}, t ; M(\bar{x}))$ for an $\bar{x}<0$. Since it is clear that $\tilde{u}_{0} \prec u_{0}$ we have $\tilde{\zeta}(t) \leq \varsigma(t)$ for every $0<t<T^{*}$. But since

$$
\tilde{\zeta}(t)=\bar{x}+c_{m} M(\bar{x})^{(m-1) /(m+1)} t^{1 /(m+1)},
$$

we conclude that $\zeta(t)>0$ if $t>c_{m}^{-(m+1)}|\bar{x}|^{m+1} M(\bar{x})^{-(m-1)}$. This being true for every $\bar{x}<0$ we can take the infimum of the expression in the right-hand side and obtain thus the desired inequality.

(I) Since, because of assumption (H2), $B$ is finite if and only if

$$
\limsup _{x \rightarrow 0} M(x)|x|^{-(m+1) /(m-1)}
$$

is finite, (3.4) follows from (3.5).

(III) We first recall that any solution $\tilde{u}(x, t)$, with initial pressure $\tilde{p}_{0}(x)=\alpha x^{2}+$ $o\left(x^{2}\right)$ and such that $p_{0}(x) \leq \alpha x^{2}$, has a waiting time given by (3.2) (Corollary A).

Now for every $\varepsilon>0$ the solution $\tilde{u}$ such that $\tilde{p}_{0}(x)=\alpha x^{2}$ if $x_{\varepsilon}<x<0$ and $\tilde{p}_{0}(x)=0$ otherwise, satisfies $\tilde{u}_{0} \prec u_{0}$ if

$$
(A-\varepsilon) \geq\left(\frac{m-1}{m+1}\right)^{1 /(m-1)} \cdot \frac{m-1}{m+1} \cdot \alpha^{1 /(m-1)}
$$

and $x_{\varepsilon}$ is small enough. Therefore $t^{*} \leq \tilde{t}^{*}=(2(m+1) \alpha)^{-1}=T_{m}(A-\varepsilon)^{1-m}$. Letting $\varepsilon \rightarrow 0$ we get (3.6).

We end the section by applying our results to a family of solutions already discussed in [5]:

EXAMPLE. We let $m=2$ and consider the solutions $u(x, t ; \theta)$ with initial data

$$
u_{0}(x)= \begin{cases}\frac{1}{2}\left[(1-\theta) \sin ^{2} x+\theta \sin ^{4} x\right] & \text { if } x \in[-\pi, 0], \\ 0 & \text { otherwise }\end{cases}
$$

with $0 \leq \theta \leq 1$. Notice that since $m=2, p_{0}(x)=2 u_{0}(x)$.

(1) We estimate the waiting time when $\theta=1$. In this case the results of [5] imply that $0.3174 \leq t^{*}$. We obtain more accurate estimates using Theorem 2: since the maximum of $M(x)|x|^{-3}$ for $-\infty<x<0$ is attained at the point $x=1.449951$ with a value $B=0.0769886$ it follows from (3.5) that

$$
0.3608 \leq t^{*} \leq 1.4432 \text {. }
$$

(2) Now we study the range of $\theta$ 's for which formula (3.2), i.e. in this case

$$
t^{*}=1 / 6(1-\theta)
$$

is valid. Since we have $M(x) x^{-3}=(1 / 6)(1-\theta)+(1 / 30)(4 \theta-1) x^{2}+O\left(x^{4}\right)$, for $x \simeq 0$, if we let $\bar{\theta}=\sup \{\theta \in[0,1]:(3.13)$ holds $\}$ we have the lower estimate $\bar{\theta} \geq 0.25$ as in [5]. But the upper estimate in (3.5) allows us to conclude that for $\theta$ near 1 , (3.13) does not hold. Indeed this happens for every $\theta \geq 0.88 \ldots$ Therefore

$$
0.25 \leq \bar{\theta} \leq 0.88 \ldots
$$

4. More self-similar solutions. To give exact rates of growth of the interface as $t \uparrow \infty$ or $t \simeq 0$ we need a suitable family of models that we construct in this section. 
For every $\alpha,-1<\alpha<2 /(m-1)$, we let $w_{\alpha}(x, t)$ be the solution of (P) with initial condition

$$
w_{\alpha}(x, 0)= \begin{cases}(\alpha+1)|x|^{\alpha} & \text { if } x<0 \\ 0 & \text { if } x \geq 0 .\end{cases}
$$

Since the map $u \mapsto \tau u$ defined by

$$
\tau u(x, t)=k u(L x, T t)
$$

where $k, L, T$ are given positive constants, transforms solutions of $u_{t}=\left(u^{m}\right)_{x x}$ into new solutions if $k^{m-1} L^{2}=T$ and since $T w_{\alpha}(x, 0)=w_{\alpha}(x, 0)$ if $k L^{\alpha}=1$ we conclude from the uniqueness of the solutions of $(\mathrm{P})$ that for every $L>0$ we have

$$
w_{\alpha}(x, t)=L^{-\alpha} w_{\alpha}\left(L x ; L^{2-\alpha(m-1)} t\right)
$$

for every $x \in \mathbf{R} t>0$. In particular, if we fix $t>0$ and then choose $L$ such that $L^{2-\alpha(m-1)} t=1$ we deduce that $w_{\alpha}$ can be represented in the form

$$
w_{\alpha}(x, t)=t^{\alpha \gamma} f_{\alpha}\left(x t^{-\gamma}\right) \quad \text { in } Q=\mathbf{R} \times(0, \infty),
$$

with $\gamma=(2-\alpha(m-1))^{-1}>0$. Therefore $w_{\alpha}$ is a self-similar solution.

It is easy to see that $f_{\alpha}(\xi)=w_{\alpha}(\xi, 1)$ is a nonnegative solution of the secondorder differential equation

$$
\left(f^{m}\right)^{\prime \prime}(\xi)=\alpha \gamma f(\xi)-\gamma \xi f^{\prime}(\xi) \quad\left({ }^{\prime}=d / d \xi\right)
$$

on the whole line $\xi \in \mathbf{R}$, such that $f(\xi)=o\left(\xi^{\alpha}\right)$ as $\xi \rightarrow \infty$ and $f(\xi)|\xi|^{-\alpha} \rightarrow(\alpha+1)$ as $\xi \rightarrow-\infty$. The fact that there exists a unique solution of (4.5) with such behaviour as $|\xi| \rightarrow \infty$ follows from the existence and uniqueness of solutions of (P).

By Property I.1 the free boundary $\varsigma_{\alpha}$ of $w_{\alpha}$ is finite. If we let

$$
\eta_{\alpha}=\varsigma_{\alpha}(1)
$$

then $0<\eta_{\alpha}<\infty$ and $\zeta_{\alpha}(t)=\eta_{\alpha} t^{\gamma}$. $\eta_{\alpha}$ depends only on $\alpha$ and $m$; in fact, remarking that $M_{\alpha}(x)=|x|^{\alpha+1} \leq(|x|+1)^{(m+1) /(m-1)}$ gives, by means of the comparison argument,

\section{PROPERTY I.1.}

$$
\eta_{\alpha} \leq T_{m}^{-\gamma}
$$

REMARKS. (1) Barenblatt [7] considered solutions of the form $t^{\delta} f\left(x t^{-\gamma}\right)$ to solve the problem

$$
\begin{cases}u_{t}=\left(u^{m}\right)_{x x} & \text { if } x>0, t>0 \\ u(x, 0)=0 & \text { if } x \geq 0 \\ u(0, t)=\sigma t^{\delta} & \text { if } t \geq 0\end{cases}
$$

This leads to the study of equation (4.5) with $\gamma=\frac{1}{2}[1+\delta(m-1)]$ and side conditions $f(0)=\sigma$ and $f(\infty)=0$. He considered the case $m>1, \delta \geq 0, \sigma>0$.

A detailed study of the problem

$$
\begin{cases}\left(f^{m}\right)^{\prime \prime}(\xi)+\gamma \xi f^{\prime}(\xi)=\delta f(\xi) & \text { for } \xi>0 \\ f(0)=U \geq 0, f(\xi) & \text { bounded as } \xi \rightarrow \infty\end{cases}
$$


with $m>1$ and independent parameters $\gamma, \delta \in \mathbf{R}$ is made by Gilding and Peletier in $[12,13]$ (where references to related works can be found). In case $U>0$ they prove that there exists a solution of (4.9) with compact support if $\gamma \geq 0$ and $2 \gamma+\delta>0$ and this solution is unique. In the particular case of (4.5), where $\alpha, \gamma, \delta$ are related as above, the conditions mean $\alpha>-2$. In this way we recover the solutions $w_{\alpha}(x, t)$ restricted to the quadrant $\{x \geq 0, t \geq 0\}$. Bounded positive solutions of (4.9) can be obtained under our conditions for $-2<\alpha \leq 0$. Since the equation (4.5) is invariant under the transformation $\eta \mapsto-\eta$ we can recover the left part, $(x \leq 0, t \geq 0)$, of $w_{\alpha}(x, t)$ if $\alpha \leq 0$.

But once we have the general existence and uniqueness theory for (P) our approach gives a very simple proof of the existence and properties of $w_{\alpha}(x, t)$ that relies on the use of the scaling-invariance of the equation.

(2) When $\alpha=(m-1)^{-1}, \gamma=1$, we obtain the explicit solution

$$
w(x, t)=\left[\frac{m-1}{m} c(c t-x)_{+}\right]^{1 /(m-1)}
$$

with a suitable $c>0$. This is called a constant-velocity front since $\zeta(t)=c t$ and $-\left(m w^{m-1} /(m-1)\right)_{x}=c$ whenever $w>0$.

(3) For $\alpha \geq(m-1)^{-1}$ the initial pressure is a convex function: $\left(w_{\alpha}(x, 0)^{m-1}\right)_{x x}$ $\geq 0$ a.e. Therefore the same holds for every $t>0$, i.e. $f^{m-1}$ is convex and the free boundary $\eta_{\alpha}(t)$ is convex.

(4) The limit case $\alpha=-1$ is represented by the solutions with finite mass, i.e. we define

$$
w_{-1}(x, t)=w(x, t ; 1) \quad \text { as in definition }(1.6)
$$

and then

$$
\eta_{-1}=c_{m} \quad \text { as in (1.8). }
$$

Using again the transformation $\tau$ we see that for $c>0$ the functions

$$
w_{\alpha, c}(x, t)=c^{2 \gamma} t^{\alpha \gamma} f\left(\bar{c}^{(m-1) \gamma} x t^{-\gamma}\right)
$$

are solutions of $(\mathrm{P})$ with inital data $w_{\alpha, c}(x, 0)=c w_{\alpha}(x, 0)$. Their interface is given by $x=\eta_{\alpha, c}(t)$, where

$$
\eta_{\alpha, c}(t)=\eta_{\alpha}\left(c^{m-1} t\right)^{\gamma}
$$

Clearly (4.14) holds also for $\alpha=-1$.

5. Behaviour for small $t$. We begin this section by showing that the behaviour of $\zeta(t)$ as $t \rightarrow 0$ depends only on the behaviour of $M(x)$ as $x \rightarrow 0$.

LEMMA 5.1. Let $u_{1}(x, t), u_{2}(x, t)$ be two solutions of $(\mathrm{P})$ with initial data $u_{1}^{0}(x)$, $u_{2}^{0}(x)$, mass functions $M_{1}(x), M_{2}(x)$ and interfaces $\zeta_{1}(t), \zeta_{2}(t)$ respectively. If

$$
\lim _{x \rightarrow 0} \frac{M_{2}(x)}{M_{1}(x)}=c, \quad 0<c<\infty,
$$

then for every $\varepsilon>0$ there exists $\tau>0$ such that if, $0<t<\tau$,

$$
\varsigma_{1}\left((c+\varepsilon)^{m-1} t\right) \geq \varsigma_{2}(t) \geq \varsigma_{1}\left((c-\varepsilon)^{m-1} t\right) .
$$


PROOF. For every $\delta>0$ there exists $x_{\delta}>0$ such that $M_{2}(x) \leq(c+\delta) M_{1}(x)$ if $x_{\delta}<x<0$. Now we use the transformation $\tau$ (cf. $\S 4$ ) on the solution $\tilde{u}_{2}$ with initial data

$$
\tilde{u}_{2}^{0}(x)= \begin{cases}u_{2}^{0}(x) & \text { if } x_{\delta}<x<0 \\ 0 & \text { otherwise. }\end{cases}
$$

We put $k=L=(1+\delta)$ so that $T=(1+\delta)^{m+1}$ and define $\tilde{u}^{*}=\tau \tilde{u}_{2}, \tilde{u}_{0}^{*}=\tau \tilde{u}_{2}^{0}$. The support of $\tilde{u}_{0}^{*}$ is contained in the interval $\left[x_{\delta}^{*}, 0\right]$, where $x_{\delta}^{*}=x_{\delta}(1+\delta)^{-1}>x_{\delta}$. Also $\tilde{M}^{*}(x) \geq \tilde{M}_{2}(x)$ for every $x$, i.e. $\tilde{u}_{0}^{*} \succ \tilde{u}_{2}^{0}$.

We now consider the solution $U(x, t)$ with initial condition

$$
U_{0}(x)= \begin{cases}u_{0}(x) & \text { if } x<x_{\delta} \\ \tilde{u}_{0}^{*}(x) & \text { if } x_{\delta}^{*}<x<0 \\ 0 & \text { otherwise }\end{cases}
$$

It is clear that $U_{0} \succ u_{2}^{0}$; hence their interfaces $Z(t), \varsigma_{2}(t)$ satisfy $Z(t) \geq \varsigma_{2}(t)$ in their common interval of definition. But since $U_{0}(x)=0$ in the interval $\left[x_{\delta}, x_{\delta}^{*}\right]$ for a certain time $\tau>0, U(x, t)$ coincides with $\tilde{u}^{*}(x, t)$ if $x>x_{\delta}$ and $0 \leq t \leq \tau$; hence $Z(t)=\tilde{\zeta}^{*}(t)$.

To prove the first inequality of (5.2) we have yet to compare $\tilde{\zeta}_{2}^{*}$ and $\zeta_{1}$. For this we use again $\tau$, now with $k=(c+\delta)(1+\delta), L=1+\delta$ and $T=(c+\delta)^{m-1}(1+\delta)^{m+1}$. We obtain a solution $\tilde{u}_{1}=\tau u_{1}$ such that

$$
\tilde{M}_{1}(x)=(c+\delta) M_{1}((1+\delta) x) \geq M_{2}((1+\delta) x) \geq \tilde{M}^{*}(x),
$$

i.e. $\tilde{u}_{1}^{0} \succ \tilde{u}^{*}$, therefore $\tilde{\zeta}_{1}(t) \geq \tilde{\zeta}^{*}(t)=Z(t) \geq \varsigma_{2}(t)$ if $0 \leq t \leq \tau$. Choosing $\delta>0$ such that $(1+\delta)^{m+1}(c+\delta)^{m-1} \leq(c+\varepsilon)^{m-1}$, this implies the desired inequality since $\tilde{\zeta}_{1}(t)=L^{-1} \zeta_{1}(T t)$.

The second inequality can be obtained by reversing the roles of $u_{1}$ and $u_{2}$.

The solutions $w_{\alpha, c}$ constructed in $\S 4$ are used to give precise growth rates for $\zeta(t)$ when $t$ is small:

THEOREM 3. Let, for some $\beta, 0 \leq \beta<(m+1) /(m-1)$,

$$
\limsup _{x \nearrow 0} M(x)|x|^{-\beta}=c
$$

with $0 \leq c \leq \infty$. Then as $t \rightarrow 0$

$$
\lambda c^{(m-1) \gamma} \leq \limsup \varsigma(t) t^{-\gamma} \leq \eta_{\alpha} c^{(m-1) \gamma},
$$

where $\alpha=\beta-1, \gamma=(2-\alpha(m-1))^{-1} ; \eta_{\alpha}($ defined in (4.6)) and $\gamma>0$ depend only on $\beta$ and $m$.

If $c$ is the limit of $M(x)|x|^{-\beta}$ as $x \uparrow 0$, then

$$
\lim _{t \rightarrow 0} \zeta(t) t^{-\gamma}=\eta_{\alpha} c^{(m-1) \gamma} .
$$

ProOF. The right-hand inequality of (5.7) and (5.8) follows from Lemma 5.1 and formula (4.14) for the interfaces of $w_{\alpha, c}$.

To prove the left-hand side of (5.7) we cbserve that there exists a sequence $x_{n} \rightarrow 0$ such that $M\left(x_{n}\right)\left|x_{n}\right|^{-\beta} \rightarrow c$ as $n \rightarrow \infty$. We may assume that $c>0$; if not 
there is nothing to prove. We consider the solutions $\bar{u}_{n}(x, t)=w\left(x-x_{n}, t ; M\left(x_{n}\right)\right)$. It is clear that for every $n, u_{0} \succ \bar{u}_{n}(x, 0)$. Therefore we have

$$
\varsigma(t) \geq \eta_{-1} M\left(x_{n}\right)^{(m-1) /(m+1)} t^{1 /(m+1)}-\left|x_{n}\right| .
$$

Now if we take a small $\varepsilon, 0<\varepsilon<c$, we have $M\left(x_{n}\right) \geq(c-\varepsilon)\left|x_{n}\right|^{\beta}$ for all large $n \geq n_{\varepsilon}$. We remark now that the function

$$
g(y)=A y^{\mu}-y, \quad 0 \leq y \leq \infty, 0<\mu<1,
$$

takes on a maximum value at $y_{\mu}=(A \mu)^{1 /(1-\mu)}$ :

$$
g\left(y_{\mu}\right)=\frac{1-\mu}{\mu} y_{\mu} .
$$

Applying this result to (5.9) with $A=\eta_{-1}(c-\varepsilon)^{(m-1) /(m+1)} t^{1 /(m+1)}$ and $\mu=$ $\beta(m-1) /(m+1)$ and setting $y_{\mu}=x_{n}$ we find that there exists a sequence $\left\{t_{n, \varepsilon}\right\}_{n}$ such that $t_{n, \varepsilon} \rightarrow 0$ as $n \rightarrow \infty$ and

$$
\varsigma\left(t_{n, \varepsilon}\right) \geq \lambda\left((c-\varepsilon)^{m-1} t_{n, \varepsilon}\right)^{\gamma},
$$

where $\lambda=\left(\beta(m-1) \eta_{-1} /(m+1)\right)^{\gamma(m+1)} \cdot(\gamma \beta(m+1))^{-1}$. Letting $\varepsilon \rightarrow 0$ we obtain the left-hand side of (5.7).

REMARKS. (1) The first results on $\zeta(t)$ for small $t$ seem to be those of [16] where it is proved that $\zeta(t)=O\left(t^{1 / 2}\right)$ if $u_{0} \in L^{\infty}(\mathbf{R})$.

The case $u_{0} \in L^{1}(\mathbf{R})$ is studied in $[\mathbf{2 0}]$ : it is proved that

$$
\varsigma(t) \leq \eta_{-1}\left(M^{m-1} t\right)^{1 /(m+1)},
$$

where $M=\left\|u_{0}\right\|_{1}$, and also that $\varsigma(t) t^{-1 /(m+1)} \rightarrow 0$ as $t \rightarrow 0$. The assumptions $u_{0} \in L^{p}(\mathbf{R}), 1<p<\infty$, are also discussed.

(2) If we let $\beta \geq 2$ in (5.6) and $c<\infty$, then $t_{*}>0$; cf. $§ 3$. If $\beta<2$ and $c>0$, then $t_{*}=0$.

(3) If $\beta=0$, the limit of $M(x)$ as $x \uparrow 0$ always exists and (5.8) applies.

6. Behaviour as $t \rightarrow \infty$. In this section we assume that $u$ is a global solution, i.e. $T^{*}=\infty$, and study the behaviour of $\zeta(t)$ for large $t$. The results parallel those of $\S 5$ but now the values of $M(x)$ as $x \rightarrow \infty$ are the only ones that matter:

LEMMA 6.1. Let $u_{1}, u_{2}$ be two global solutions of $(\mathrm{P})$ with initial data $u_{1}^{0}, u_{2}^{0}$, mass functions $M_{1}, M_{2}$, and interfaces $\zeta_{1}, \zeta_{2}$ respectively. If

$$
\lim _{x \rightarrow-\infty} \frac{M_{2}(x)}{M_{1}(x)}=c, \quad 0<c<\infty,
$$

then for every $\varepsilon>0$ there exists $C_{\varepsilon}>0$ such that

$$
\varsigma_{1}\left((c+\varepsilon)^{m-1} t\right)+C_{\varepsilon} \geq \varsigma_{2}(t) \geq \varsigma_{1}\left((c-\varepsilon)^{m-1} t\right)-C_{\varepsilon} .
$$

PROOF. For every $\varepsilon>0$, there exists $x_{\varepsilon}$ such that, for $x<x_{\varepsilon}<0, M_{2}(x) \leq$ $(c+\varepsilon) M_{1}(x)=\tilde{M}_{1}(x)$, where $\tilde{M}_{1}$ is the mass of $\tilde{u}_{1}=\tau u_{1}$ and the constants in the transformation are $k=c+\varepsilon, L=1, T=(c+\varepsilon)^{m-1}$. Therefore we have, for every $x$,

$$
M_{2}(x) \leq \tilde{M}_{1}\left(x+x_{\varepsilon}\right)
$$


It follows that

$$
\zeta_{2}(t) \leq \tilde{\zeta}_{1}(t)+\left|x_{\varepsilon}\right|=\varsigma_{1}\left((c+\varepsilon)^{m-1} t\right)+\left|x_{\varepsilon}\right| .
$$

Putting $C_{\varepsilon}=\left|x_{\varepsilon}\right|$ we obtain the first inequality. The second is similar.

REMARKS. (1) As in preceding sections Knerr [16] obtained the first results: Under simplifying assumptions on the initial data (cf. §3) he proved that $\varsigma(t)=$ $O\left(t^{1 /(m+1)}\right)$. In [20] very precise results are obtained when $u_{0} \in L^{1}(\mathbf{R})$ (and satisfies (H1), (H3)): it is proved that

$$
\begin{aligned}
& \text { (i) } \varsigma(t) t^{-1 /(m+1)} \rightarrow c_{m} M^{(m-1) /(m+1)}, \quad M=\left\|u_{0}\right\|_{1}, \\
& \text { (ii) } \varsigma^{\prime}(t) t^{m /(m+1)} \rightarrow \frac{c_{m}}{(m+1)} M^{(m-1) /(m+1)}, \quad \text { and } \\
& \text { (iii) } \varsigma(t)-c_{m} M^{(m-1) /(m+1)} t^{1 /(m+1)} \downarrow x_{0}=M^{-1} \int x u_{0}(x) d x .
\end{aligned}
$$

$x_{0}$, the center of mass, can be finite or $-\infty$. Notice that (6.5) implies that, for every solution, $\varsigma(t)$ grows at least like $t^{1 /(m+1)}$.

Using the solutions $w_{\alpha, c}$ in combination with Lemma 6.1 we obtain the following growth rates:

THEOREM 4. Let, for some $\beta, 0 \leq \beta<(m+1) /(m-1)$,

$$
\limsup _{x \rightarrow-\infty} M(x)|x|^{-\beta}=c
$$

with $0 \leq c \leq \infty$. Then as $t \rightarrow \infty$

$$
\lambda c^{(m-1) \gamma} \leq \limsup \varsigma(t) t^{-\gamma} \leq \eta_{\alpha} c^{(m-1) \gamma},
$$

where $\alpha, \gamma, \lambda$ and $\eta_{\alpha}$ are as in Theorem 3. If, moreover, $c$ is the limit of $M(x)|x|^{-\beta}$ as $x \rightarrow-\infty$, then

$$
\lim _{t \rightarrow \infty} \varsigma(t) t^{-\gamma}=\eta_{\alpha} c^{(m-1) \gamma} .
$$

PROOF. It is completely similar to that of Theorem 3 , only changing throughout $t \downarrow 0, x \uparrow 0$ into $t \rightarrow \infty, x \rightarrow-\infty$.

REMARKS. (2) If we allow $\beta=2$ in (6.6) then if $c>0$ there is a blow-up in finite time. In case $\beta>2, c>0$, no solution of $(\mathrm{P})$ exists. If $\beta=0$ the limit of $M(x)$ as $x \rightarrow-\infty$ always exists, finite or infinite.

(3) The case $u_{0} \in L^{p}(\mathbf{R}), 1 \leq p \leq \infty$, is treated in [20]. Notice that $u_{0} \in L^{p}(\mathbf{R})$ implies $M(x)=O\left(|x|^{\beta}\right)$ with $\beta=(p-1) / p$ if $p<\infty, \beta=1$ if $p=\infty$.

7. Approaching a blow-up. In this section we assume that the blow-up time $T^{*}$ is finite, i.e. that $M(x)|x|^{-(m+1) /(m-1)}$ does not tend to 0 as $x$ goes to $-\infty$. We begin by describing the different possible behaviours of the interface $\varsigma(t)$ as $t \uparrow T^{*}$. Let

$$
l^{*}=\lim _{t \uparrow T^{*}} \varsigma(t), \quad v^{*}=\lim _{t \uparrow T^{*}} \varsigma^{\prime}(t) .
$$

Both limits exist, either finite or infinite. The four cases that may occur are:

(I) $l^{*}=0$, i.e. $t^{*}=T^{*}$ and $\varsigma(t)=0$ for $0 \leq t \leq T^{*}$. Example: $z_{-}\left(x, t ; T^{*}\right)$.

(II) $0<l^{*}<\infty, 0<v^{*}<\infty$.

(III) $0<l^{*}<\infty, v^{*}=\infty$. Example: $z_{-}\left(x, t ; T^{*}, C\right)$ with $C<0$. 
(IV) $l^{*}=\infty, v^{*}=\infty$

Remark that because of (1.15) $l^{*}>0$ implies $v^{*}>0$ and $l^{*}=\infty$ implies $v^{*}=\infty$.

An example of type (II) is easily constructed as follows: let $u$ be the solution with initial data

$$
u_{0}(x)=z_{-}(x+1,0 ; T)+M \delta(x),
$$

where $M, T>0$. If $M$ and $T$ are small enough, $u$ equals exactly $z_{-}(x+1, t ; T)+$ $w(x, t ; M)$ in $Q_{T}$, has blow-up time $T$ and $\zeta(t)=r_{M}(t)$ for $0<t<T$.

On the other hand it follows from Theorems 1 and 2 that (I) happens when the limit of $M(x)|x|^{-(m+1) /(m-1)}$ exists as $x \rightarrow-\infty$ and equals $B$.

Examples of type (IV) will follow from Proposition 7.1.

We introduce now a useful concept, that of blow-up set $\Sigma=\Sigma(u)$ :

$$
\Sigma=\left\{x \in \mathbf{R}: u(x, t) \rightarrow \infty \text { as } t \rightarrow T^{*}\right\} .
$$

Note that the limit of $u(x, t)$ as $t \uparrow T^{*}$ exists for every $x \in \mathbf{R}$ since $u_{t} \geq-u /(m+1) t$, i.e. $u(x, t) t^{1 /(m+1)}$ is nondecreasing in $t$, in $Q_{T^{*}}$. The following holds:

Proposition 7.1. $\Sigma$ is an interval beginning at $-\infty$.

Proof. Let $\bar{x}$ be a point not belonging to $\Sigma$. For simplicity we take $\bar{x}=0$. Since $\lim u(0, t)<\infty$ as $t \uparrow T^{*}$ there exists $C>0$ such that $u(0, t) \leq C$ for $T^{*} / 2 \leq t<T^{*}$. We want to prove that no $x>0$ belongs to $\Sigma$. This is obvious if $l_{*}=0$; hence in the sequel we assume that $\zeta(t)>0$ for $t \geq T^{*}-\varepsilon>T^{*} / 2$.

Consider for $T^{*}-\varepsilon<t<T^{*}$ the function $p(x)=m u^{m-1}(x, t) /(m-1), p$ is a continuous nonnegative function on the interval $(0, \varsigma(t))$ such that $p(0) \leq$ $C, p(\varsigma(t))=0$ and $p_{x x} \geq-K$, where $K=\left((m+1) T^{*} / 2\right)^{-1}$. Now we take the parabola $\bar{p}(x)=\alpha-(K / 2)(x-\beta)^{2}$ that passes through $(0, C)$ and $(\zeta(t), 0)$, i.e. with

$$
\alpha=\frac{K}{2}\left(\frac{\zeta}{2}+\frac{C}{K_{\zeta}}\right)^{2}, \quad \beta=\frac{\zeta}{2}-\frac{C}{K \zeta} .
$$

It is easy to see that $p(x) \leq \bar{p}(x)$ in $(0, \varsigma(t))$; hence, in particular,

$$
\zeta^{\prime}(t)=-p_{x}(\zeta(t)) \leq-\bar{p}_{x}(\zeta(t))=\left(K_{\zeta}(t) / 2+C_{\zeta}(t)^{-1}\right) .
$$

Integrating (7.5) from $T^{*}-\varepsilon$ we conclude that $\zeta(t), \zeta^{\prime}(t)$ remain finite as $t \uparrow T^{*}$. Since the maximum of $p(x, t)$ in $x$ in $[0, \zeta(t)]$ is less than $\alpha$, and $\alpha$ is bounded for $t \rightarrow T^{*}$ we conclude that for every $x>0, x \notin \Sigma$.

We set $b^{*}=\sup \Sigma$. It is clear that $-\infty \leq b^{*} \leq l^{*}$. Moreover, from the above proof follows

COROLlaRY 7.1. If $b^{*}$ is finite, then $l^{*}$ is finite. If also $l^{*}>b^{*}$, then $v^{*}$ is finite.

When $u_{0}$ is nicely behaved at $-\infty$ there is a simple formula for $b^{*}$. Indeed if we assume that the limits

$$
\lim _{x \rightarrow-\infty} M(x)|x|^{-(m+1) /(m-1)}=L_{0}, \quad 0<L_{0}<\infty,
$$

and

$$
\lim _{x \rightarrow-\infty}\left(\left(\frac{M(x)}{L_{0}}\right)^{(m-1) /(m+1)}+x\right)=c, \quad-\infty \leq c \leq+\infty
$$


exist, then we have

PROPOSITION 7.2. $b^{*}=c$.

Proof. (i) $b^{*} \leq c$. Assume that $c<\infty$. We shall prove that for every $c^{\prime}>$ $c, b^{*} \leq c^{\prime}$. In fact, there exists $C_{1}>0$ such that $M(x) \leq L_{0}\left(-x+c^{\prime}\right)^{(m-1) /(m+1)}$ for every $x<-C_{1}$. This means that by virtue of Property S3,

$$
\int_{-\infty}^{\infty}\left(u(x, t)-z_{-}\left(x-c^{\prime}, t ; T^{*}\right)\right)_{+} d x \leq N^{\prime}<\infty
$$

for every $0<t<T^{*}$, where $N^{\prime}$ does not depend on $t$. Now since $z_{-}\left(x-c^{\prime}, t ; T^{*}\right)=0$ for $x \geq c^{\prime}$ we deduce that

$$
\int_{c^{\prime}}^{\infty} u(x, t) d x \leq N^{\prime}, \quad 0<t<T^{*} .
$$

This implies that $c^{\prime} \geq b^{*}$ because of the following lemma. Hence $b^{*} \leq c$.

LEMMA 7.1. For every $x_{0}<b^{*}$ and every $\varepsilon>0$ we have

$$
\lim _{t \rightarrow T^{*}} \int_{\left|x-x_{0}\right|<\varepsilon} u(x, t) d x=\infty .
$$

PROOF. Since $\left(u^{m-1}\right)_{x x} \geq-K$ (see proof of Proposition 7.1) we have, for every $x, x_{0} \in \mathbf{R}, u(x, t) \geq u\left(x_{0}, t\right)-(K / 2)\left(x-x_{0}\right)^{2}$ either for $x>x_{0}$ or for $x<x_{0}$. Hence

$$
\int_{\left|x-x_{0}\right|<\varepsilon} u(x, t) d x \geq\left(u\left(x_{0}, t\right)-\frac{k}{2} \varepsilon^{2}\right) \varepsilon .
$$

As $u\left(x_{0}, t\right) \uparrow \infty$ as $t \uparrow T^{*}$ the result follows.

(ii) $b^{*} \geq c$. Arguing as above if $c>-\infty$, for every $c^{\prime \prime}<c$ there exists $N^{\prime \prime}>0$ such that

$$
\int_{-\infty}^{\infty}\left(z_{-}\left(x-c^{\prime \prime}, t ; T^{*}\right)-u(x, t)\right)_{+} d x \leq N^{\prime \prime}<\infty .
$$

Now if $b^{*}<c^{\prime \prime}$ this implies, arguing as in Proposition 7.1, that $u(x, t)$ is bounded above in $\left(c^{\prime \prime \prime}, c^{\prime \prime}\right) \times\left(T^{*} / 2, T^{*}\right)$ for any $c^{\prime \prime \prime} \in\left(B^{*}, c^{\prime \prime}\right)$. However, Lemma 7.1 applied to $z_{-}$implies that as $t \rightarrow T^{*}$

$$
\int_{b^{*}}^{c^{\prime \prime}} z_{-}\left(x-c^{\prime \prime}, t ; T^{*}\right) d x \rightarrow \infty
$$

contradicting (7.10). Therefore $b^{*} \geq c^{\prime \prime}$, hence $b^{*} \geq c$.

Under the above assumptions the type (IV) corresponds precisely to $c=+\infty$. If $c<0$ we have an interface of type (I) or (II): remark that in this case we can replace the lim in condition (H4) by lim sup (and the same proof implies that $b^{*} \leq c<0=l^{*}$ ). We remark finally that when $c=-\infty$ the blow-up set $\Sigma$ is void: in this case the sequence

$$
s_{n}=\sup _{x<-1}|x|^{-(m+1) /(m-1)} \int u\left(x, t_{n}\right) d x
$$

must diverge as $t_{n} \rightarrow T^{*}$ but the sup is taken at points $x_{n} \rightarrow-\infty$. 
It would seem that the blow-up merely concern the set $\Sigma$. However, the next result points out a global aspect:

Consider a solution $u$ with initial data $u_{0}$ that blows up at time $T>0$. Let $\left\{u_{0 n}\right\}$ be an increasing sequence of measures that converge to $u_{0}$ and let $\left\{u_{n}\right\}$ be the corresponding solutions. Let $\zeta, \zeta_{n}$ be their respective free boundaries. We choose $u_{0 n}$ so that $u_{n}$ exists for all time $0<t$. We have

Proposition 7.3. (i) For every $(x, t) \in Q_{T}, u_{n}(x, t) \uparrow u(x, t)$.

(ii) For every $0<t<T, \zeta_{n}(t) \uparrow \varsigma(t)$.

(iii) For every $t>T, \zeta_{n}(t) \uparrow \infty$ and $\zeta_{n}^{\prime}(t) \uparrow \infty$.

(iv) For every $x \in R$ and $t>T, u_{n}(x, t) \uparrow \infty$.

PROOF. (i) It is clear from the maximum principle that for every $n, u_{n} \leq$ $u_{n+1} \leq u$ whenever they are defined. Theorem E and Proposition 1.6 of [9] prove that the sequences $\left\{u_{n}^{m}\right\},\left\{\left(u_{n}^{m}\right)_{x}\right\}$ and $\left\{\left(u_{n}^{m}\right)_{t}\right\}$ are uniformly bounded in $L_{\text {loc }}^{2}\left(\mathbf{R} \times(0, T)\right.$. Hence $\left\{u_{n}^{n}\right\}$ converges uniformly on compacts to a continuous solution $\bar{u}$ of $u_{t}=\left(u^{m}\right)_{x x}$ in $Q_{T}$. Its initial trace (that exists by [4]) $\bar{u}_{0}$ necessarily satisfies $u_{0 n} \leq \bar{u}_{0}$. Moreover, $\bar{u} \leq u$. It follows from Theorem B that $u=\bar{u}$.

(ii) Since, obviously, $\zeta_{n}(t) \leq \zeta_{n+1}(t) \leq \zeta(t)$ whenever they are defined, we have to prove that, for $t<T, \lim _{n} \zeta_{n}(t) \equiv \sigma(t) \geq \varsigma(t)$. In fact, if $\sigma\left(t_{0}\right)=\varsigma\left(t_{0}\right)-\varepsilon$, for some $t_{0}<T$ and $\varepsilon>0$, this can only happen at a point where $\zeta$ already moves: $\varsigma^{\prime}\left(t_{0}\right)=$ $k>0$. Using the fact that $\varsigma^{\prime}\left(t_{0}\right)=-p_{x}\left(\zeta\left(t_{0}\right) ; t_{0}\right)$, where $p=(m /(m-1)) u^{m-1}$, we conclude that $u\left(x, t_{0}\right)>0$ for $x$ near $\zeta\left(t_{0}\right)$. Since $u_{n}\left(x, t_{0}\right)=0$ for every $x \geq \varsigma_{n}\left(t_{0}\right) \leq \sigma\left(t_{0}\right)$ we arrive at a contradiction with (i).

(iii) This is the first interesting point. We know (Theorem $\mathrm{A}(\mathrm{iv}))$ that \|\|$u(\cdot, t)\|\|_{1}$ $\rightarrow \infty$ as $t \rightarrow T$. Since $u_{n} \uparrow u$ it follows that for every $n$ there exist an integer $j_{n}$ and a point $\left(x_{n}, t_{n}\right) \in Q_{T}$ such that

$$
\int_{x_{n}}^{\infty} u_{j_{n}}\left(x, t_{n}\right) d x \geq\left(n\left|x_{n}\right|\right)^{(m+1) /(m-1)}, \quad t_{n} \rightarrow T, x_{n}<-1 .
$$

We consider now the solution $\bar{u}_{n}(x, t)=w\left(x-x_{n}, t-t_{n} ; n\left|x_{n}\right|^{(m+1) /(m-1)}\right)$, defined for $t \geq t_{n}$. By Corollary S1 we have, since $u_{j_{n}} \succ \bar{u}_{n}$ at time $t_{n}$,

$$
\zeta_{j_{n}}(t) \geq c_{m}\left(n\left|x_{n}\right|\right)\left(t-t_{n}\right)^{1 /(m+1)}-x_{n} \text { if } t>t_{n} .
$$

Now fix $t=T+\tau>T$ and let $n \rightarrow \infty$ in (7.14) to obtain $\zeta_{n}(t) \uparrow \infty$.

From (1.15) it follows that, for every $t>0, \zeta_{n}^{\prime}(t) t \geq(m+1)\left(\zeta_{n}(t)-\zeta_{n}(0)\right)$. Hence if $t>T$ and $n \rightarrow \infty, \zeta_{n}^{\prime}(t) \rightarrow \infty$.

(iv) Let $p_{n}(x, t)=(m /(m-1)) u_{n}^{m-1}$. Since $p_{n} \geq 0,\left(p_{n}\right)_{x x} \geq-((m+1) t)^{-1}$ and $\left(p_{n}\right)_{x}\left(\zeta_{n}(t), t\right)=-\zeta_{n}^{\prime}(t)$ it follows from (iii) that, for $t>T, \lim u_{n}\left(\zeta_{n}(t)-1, t\right)=\infty$ as $n \rightarrow \infty$. The conclusion $u_{n}(x, t) \uparrow 0$ for every $x>0$ follows from the fact that $u_{n}$ is nondecreasing in $x$ for $x>0, t>0$. A proof of this property using Caffarelli's Reflection Principle is as follows: If we compare the functions $u_{n}(x, t)$ and $\bar{u}_{n}(x, t)=u_{n}(2 a-x, t)$ in a domain $D=(a, \infty) \times(0, \infty)$ with $a \geq 0$, it follows from the maximum principle that $u_{n} \leq \bar{u}_{n}$. Now, given $0 \leq x_{1}<x_{2}$ and $t>0$, take $a=1 / 2\left(x_{1}+x_{2}\right)$ to conclude that $u_{n}\left(x_{1}, t\right) \geq u_{n}\left(x_{2}, t\right)$.

To prove that $u_{n}(x, t) \uparrow 0$ even for $x<0$ we consider the solutions $\hat{u}_{n}$ with initial data $\hat{u}_{0 n}=u_{0 n} \cdot \chi(-\infty, a)$ with $a<0$. They approximate the solution $\hat{u}$ with $\hat{u}_{0}=u_{0} \cdot \chi(-\infty, a)$. Since $\hat{T}=T$ we apply the above to conclude that $\hat{u}_{n}(x, t) \uparrow \infty$ for every $x \geq a, t>T$. But $\hat{u}_{n} \leq u_{n}$. 
8. Other interfaces. If $u$ is a solution of (P) under conditions (H1)-(H3) and $u_{0}(x)=0$ for $x \leq a$, then an outer left-interface appears:

$$
\text { Sleft }(t)=\inf \{x: u(x, t)>0\}, \quad t>0 .
$$

The properties of $\zeta_{\text {left }}$ are completely similar to those of $\zeta(t)$. Since $u_{0} \in L^{1}(\mathbf{R})$, the asymptotic behaviour as $t \rightarrow \infty$ is covered in [20].

Also an inner free boundary $\Gamma_{\text {in }}$ may appear: it is the part of the boundary $\Gamma$ of $\Omega=\left\{(x, t) ; x \in \mathbf{R}, 0<t<T^{*}\right.$ and $\left.u(x, t)>0\right\}$ in $Q_{T}^{*}$ not contained in $x=\varsigma(t)$ or $x=\varsigma_{\text {left }}(t)$. As explained in [20] it consists of an at most countable number of locally Lipschitz arcs beginning at $t=0$. Cf. [20] for other details.

ACKNOWLEDGEMENTS. The author is indebted to the University of Minnesota and the Madison Research Center for their hospitality. He is particularly grateful to D. G. Aronson for many discussions and suggestions and to C. E. Kenig who kindly provided some unpublished results.

\section{REFERENCES}

1. D. G. Aronson, Regularity properties of flows through porous media: the interface, Arch. Rational Mech. Anal. 37 (1970), 1-10.

2. $\ldots$, Regularity properties of flows through porous media: a counterexample, SIAM J. Appl. Math. 19 (1970), 299-307.

3. D. G. Aronson and $\mathrm{Ph}$. Bénilan, Régularité des solutions de l'équation des millieux poreux dans $\mathbf{R}^{n}$, C.R. Acad. Sci. Paris 288 (1979), 103-105.

4. D. G. Aronson and L. A. Caffarelli, The initial trace of a solution of the porons medium equation, Trans. Amer. Math. Soc. 280 (1983), 351-366.

5. D. G. Aronson, L. A. Caffarelli and S. Kamin, How an initially stationary interface begins to move in porous medium flow, SIAM J. Math. Anal. 14 (1983), 639-658.

6. D. G. Aronson, L. A. Caffarelli and J. L. Vazquez, Interfaces with a corner point in one-dimensional porous medium flow, preprint.

7. G. I. Barenblatt, On some unsteady motions of a liquid or a gas in a porous medium, Prikl. Mat. Mekh. 16 (1952), 67-78. (Russian)

8. Ph. Bénilan and M. G. Crandall, The continuous dependence on $\phi$ of the solutions of $u_{t}-\Delta \phi(u)=$ 0, Indiana Univ. Math. J. 30 (1981), 161-177.

9. Ph. Bénilan, M. G. Crandall and M. Pierre, Solutions of the porous medium equation in $\mathbf{R}^{n}$ under optimal conditions on initial values, Indiana Univ. Math. J. 33 (1984), 51-87.

10. L. A. Caffarelli and A. Friedman, Regularity of the free boundary for the one-dimensional flow of gas in a porous medium, Amer. J. Math. 101 (1979), 1193-1218.

11. B. E. J. Dahlberg and C. E. Kenig, Non-negative solutions of the porous medium equation, Comm. Partial Differential Equations (to appear).

12. B. H. Gilding and L. A. Peletier, On a class of similarity solutions of the porous media equation, J. Math. Anal. Appl. 55 (1976), 351-364.

13. __ On a class of similarity solutions of the porous media equation. II, J. Math. Anal. Appl. 57 (1977), 522-538.

14. A. S. Kalashnikov, the Cauchy problem in the class of increasing functions for equations of unsteady filtration type, Vestnik Moskov. Univ. Ser. VI Mat. Mekh. 6 (1963), 17-27. (Russian)

15. A. A. Lacey, J. R. Ockendon and A. B. Tayler, 'Waiting-time' solutions of a nonlinear duffusion equation, SIAM J. Appl. Math. 42 (1982), 1252-1264.

16. B. F. Knerr, The porous medium equation in one dimension, Trans. Amer. Math. Soc. 234 (1977), 381-415.

17. O. A. Oleinik, A. S. Kalashnikov and Czhou Yui Lin, The Cauchy problem and boundary problems for equations of the type of nonstationary filtration, Izv. Akad. Nauk SSSR Ser. Mat. 22 (1958), 667-704. (Russian)

18. L. A. Peletier, A necessary and sufficient condition for the existence of an interface in flows through porous media, Arch. Rational Mech. Anal. 56 (1974), 183-190. 
19. P. E. Sacks, Continuity of solutions of a singular parabolic equation, Nonlinear Anal. 7 (1983), 387-409.

20. J. L. Vazquez, Asymptotic behaviour and propagation properties of the one-dimensional flow of gas in a porous medium, Trans. Amer. Math. Soc. 277 (1983), 507-527.

División MATEmáticas, Universidad Autónoma, Madrid 34, SPAin (Current address) SChool of MATHematics, University of Minnesota, Minneapolis, Minnesota 55455 